\title{
Large Surveys in Cosmology: The Changing Sociology
}

\author{
Ofer Lahav, Institute of Astronomy, Madingley Road, Cambridge CB3 0HA, UK \\ E-mail: lahav@ast.cam.ac.uk
}

October 30, 2018

\begin{abstract}
Galaxy redshift surveys and Cosmic Microwave Background experiments are undertaken with larger and larger teams, in a fashion reminiscent of particle physics experiments and the human genome projects. We discuss the role of young researchers, the issue of multiple authorship, and ways to communicate effectively in teams of tens to hundreds of collaborators. 1]
\end{abstract}

\section{Introduction}

The field of Observational Cosmology is going through an 'inflationary phase'. Galaxy redshift surveys will soon register millions of galaxies, imaging surveys will soon record Terabytes per observing night, and the Cosmic Microwave Background experiments will map the sky at very high resolution and sensitivity.

These technological developments have immediate effects on the human interaction. We commonly hear at conferences statements like "to make a big impact in Astronomy, a new survey must be 10-1000 times better in sensitivity and/or resolution and/or the number of objects", and "it takes at least 2-3 times the most pessimistic estimate to begin/complete/analyse a survey". This indicates that conducting large surveys in this competitive field is becoming more demanding, and that the new technology heavily relies on human resources. While improving the technology of the big surveys, the human aspects should not be neglected. This raises some questions about the changing sociology of doing research in modern Astronomy:

- What is the individual's contribution in a big collaboration (cf. the particle physics experiments and the human genome project ) ?

- How to communicate effectively (via e-mail, web, meetings) in a team of 20-200 collaborators?

- What skills should be acquired by the next generation of Astronomers ?

- Will the increase in projects and data sets be followed by more jobs for young astronomers?

\footnotetext{
${ }^{1}$ Invited article for 'Organizations and Strategies in Astronomy II', Ed. Andre Heck
} 
- How should the community deal with public domain data (e.g. HDF and the proposed Virtual Observatories)?

- How to communicate the knowledge resulting from the surveys to the tax-payer ?

Here we examine some of these issues. I happen to be involved in several large collaborations, and I have chosen to illustrate some of the points by using as an example the ongoing 2-degree-Field Galaxy Redshift Survey (2dFGRS). Needless to say, the points made below are my own views, and they do not necessarily represent a 'party line'.

\section{Large surveys, large collaborations}

Astronomy has been unique among the sciences in allowing small teams (of 3-8 people) to compete for time on world-class telescopes. Although the telescopes are built by hundreds of people, time is allocated (via competition) among the community at large, and only those involved in the science appear on the resulting paper (with the facility acknowledged and the instrument builders referenced). This made Astronomy more attractive to some young researchers than say particle physics, where papers include hundreds of authors. However, the new big surveys in Astronomy require many more participants.

We shall first discuss the big redshift surveys. Multi-fibre technology now allows us to measure redshifts of millions of galaxies. Two major surveys are underway. The US Sloan Digital Sky Survey (SDSS 27) will measure redshifts of about 1 million galaxies over a quarter of the sky. The Anglo-Australian 2 degree Field $(2 \mathrm{dF})$ survey ${ }^{3}$ will measure redshifts for 250,000 galaxies selected from the APM catalogue. Over 150,000 2dF redshifts have been measured so far (as of April 2001).

By the standards of the new millennium the 2dFGRS is a medium-sized collaboration (20-30 people). For reference, the QSO 2dF survey thas only 6 collaborators. On the other hand, the ambitious Sloan Digital Sky Survey (SDSS) involves a formal list of roughly 100 'builders', people who have put at least 2 years effort into the project (York et al. 2000). The SDSS home page on collaboration mentions 150 scientists at the 11 participating institutions, but there are about 400 people with data rights. The SDSS is coordinated by a Collaboration Council ('CoCo') which helps to manage the affairs of the collaboration. Examples of other large 'ground-based' collaborations are microlensing experiments (e.g. Machos) and imaging surveys (e.g. Vista, which involves 18 universities in the UK).

The Cosmic Microwave Background (CMB) experiments also require large collaborations with new management strategies. While recent and ongoing ex-

\footnotetext{
${ }^{2}$ http://www.sdss.org/

${ }^{3}$ http://www.mso.anu.edu.au/2dFGRS/

${ }^{4}$ http://www.2dfquasar.org/

${ }^{5}$ http://www.sdss.org/collaboration/index.html
} 
periments like Boomerang 1 , Maxima 7 and MAP \$ have 'only' 20-40 collaborators each, the Planck project is of a different order of magnitude.

In the Planck project of (to be launched in 2007) there are several degrees of involvement (without counting the people involved from industry). There are 50-100 people on the management level, some 100-200 people involved with the instrumentation, and at least 30 people who are involved with data analysis on a day-to-day basis 10 .

When it comes to considering the optimal size of a collaboration, it is worth recalling some remarks from the autobiography of Fred Hoyle (1994): "The essential point - the overriding point- is that the number of people with whom we need to interact in our daily lives should not exceed about one hundred, and preferably, on any enterprise of difficulty, not more than twenty-five. This is because twenty-five was the typical size of the hunting parties of pre-history. It is the scale of the medieval village, the scale of the modern cabinet in government, ... More or less everything that lies within it will be successful, and more or less everything that lies outside it will not".

\section{$3 \quad 2 \mathrm{dFGRS}$ as a test case}

The 2dFGRS (e.g. Folkes et al. 1999; Peacock et al. 2001) includes about 20 core members and in addition about 10 students and post-docs who are heavily involved in the survey. Most of the collaborators are in various institutions in the UK and in Australia, so there are in fact two sub-teams, with principal investigators in each of the countries. This geographical distribution has led to regular 'half-team' 1-day meetings (about 2-3 per year) in the two countries, and to regular Email/WWW exchange. The 2dFRGS group web-site has proved useful for exchanging data, results, and minutes of meetings among the team members.

Since the time the 2dFGRS team formed (around 1995), some members have left and others have joined. Also, the scientific goals have somewhat changed given the rapid progress in other areas of Cosmology (e.g. the CMB). This requires frequent updates on 'who is doing what', with careful attention to protecting the work of $\mathrm{PhD}$ students and post-docs.

The 2dFGRS is not complete yet, so it is too early to assess its overall performance, but on the whole one can make the following observations:

* It has taken some time for all involved to get used to the 'loss of individuality' and to the structure of the big collaboration, to agree on the division of labour, and to develop appropriate communication channels.

* The regular meetings in the UK and in Australia are very useful in focusing attention on technical issues and progress with papers.

\footnotetext{
${ }^{6}$ http://www.physics.ucsb.edu/ boomerang/team.html

${ }^{7}$ http://cfpa.berkeley.edu/group/cmb/maximapeople.html

${ }^{8}$ http://map.gsfc.nasa.gov/html/institutions.html

${ }^{9}$ http://astro.estec.esa.nl/Planck/

${ }^{10}$ For the big projects like SDSS and Planck it was actually difficult to find accurate estimates for the number of people involved. This is by itself an interesting fact.
} 
* The Email/WWW exchange is quite efficient, even if daunting at times (see below).

* Decisions on papers and authorship have usually reached reasonable agreement after iterations among the relevant people.

* Requests for external collaborators have been dealt with in a democratic way, by consulting the entire team.

\section{The role of $\mathrm{PhD}$ students and Post-Docs}

Young researchers may find themselves in big collaborations, co-authoring papers with tens of authors, and with collaborators they have never met. Although there is a danger that individuals (junior as well as senior) might be 'lost in the crowd', there are some benefits for a young person to be involved in a collaboration at the forefront of research. However, it is crucial to identify a niche, which is not already taken by other senior members of the collaborations, or by other students. When a PhD student or a post-doc has such a territory, his/her work, if of high quality, is recognized by a large number of people well ahead of publication. There is also a constant exchange of ideas and cross-fertilization with collaborators who are leaders of the field. This means that the young person can get exposure and can form a international 'network' at an early stage of his/her career. Being appreciated by a number of senior people also improves the chances of getting post-doctoral and faculty positions.

There is however, at least one problematic issue related to long term projects. Some post-docs are employed for a period of 2-3 years primarily to develop algorithms and pipe-line software for future experiments. This means that when they next apply for jobs, it would be difficult for them to present 'real' scientific output. The 'reward system' for those who put in several years of hard work on technical aspects of the surveys varies from country to country and from one institution to another. Is some places more can be done to improve it.

There are a number of solutions to this problem (heard occasionally in Cambridge pubs):

* To assign some of the development task to national laboratories, where PhDs with permanent positions can develop long term projects without the worry of 'publish or perish'.

* To enable post-docs who are working on software development etc. to spend say $50 \%$ of their time on science of their choice.

* To change the rules of assessment for positions from being based entirely on listed journal papers to other products such as software packages or management achievements. There should be career paths for such people which are as highly regarded as the standard academic university track. This assessment would of course heavily depend on references from senior members of the collaboration.

Another aspect of the large surveys is that the skills required for some of the tasks are quite different from the $\mathrm{PhD}$ qualifications of the previous generation. While there is still great need for post-docs and students with analytic skills and deep knowledge of the Landau \& Lifshitz volumes, we see a new generation 
of successful post-docs who have stronger emphasis on numerical and computational work. The group dynamic of the large collaborations also suggests that those with good communication skills have better chances of succeeding.

It remains to be seen if the growth in projects and 'soft-money' positions will eventually lead to more tenured positions in Astronomy. This issue is beyond the scope of this article, but it is clear that the probability of a young researcher eventually getting a permanent position also affects the research patterns in the large collaborations.

\section{Email traffic and the WWW}

Joining big teams also means spending a large fraction of the day on Email. The Email and the World Wide Web (WWW) media make the interaction between people in different institutions and countries easier, but it consumes time and energy. The ethics of using Email have not yet been structured in the society (see e.g. Wallace 1999), and one can experience daily different style of Email communications.

In a large collaboration most of the Email messages circulated are relevant to only a subset of the team. One may choose to send an email only to a subset of the team, but then others may get upset about not being informed! I found it helpful when a message circulated to the entire team gives in 1-2 sentences at the top a summary of the main point, with clear indication of who might be directly interested in it, and who is expected to act upon it. It is also helpful if the sender points to material such as tables and plots in his/her web home page, instead of sending huge files to the Email-boxes of numerous collaborators.

In the SDSS collaboration there are different (about 40 in total) of Email exploders for all aspects of SDSS (e.g. photometric pipeline, galaxy science, etc.), which are archived on the web. This allows people to choose to pay attention to just those aspects they find important.

Other problematic issues related to email are well known, e.g. misunderstanding over language, style and terminology. For example, from time to time messages with sensitive 'political' issues make it (by chance or by design !) to those who were not supposed to see them. In certain circumstances it is worth remembering to use, instead of Email, the good old telephone!

\section{Multiple Authorship}

We examined the number of authors in volumes of the Astrophysical Journal (ApJ). Of the 32 papers published in the first volume of the ApJ in 1895, 31 $(97 \%)$ were written by a single author. On the other hand, in the first volume of ApJ 2000, only $15 \%$ were written by a single author, $70 \%$ written by $2-5$ authors, and $15 \%$ written by 6 and more authors.

The recent 2dFGRS papers (e.g. Folkes et al. 1999; Peacock et al. 2001) have about 25-30 authors. This long list of authors attempts to reflect the division 
of labour regarding instrumentation, observations, data reduction, analysis and theory. The author-list is usually led by the 5-6 authors who contributed most to that particular paper, and the rest are listed by alphabetic order. The credit for people is complicated even more by the fact that the big surveys are stretched over many years, so some participants leave the project (or quit Astronomy) and others join in.

In the SDSS collaboration there are formal rules about authorship, e.g. a first list of people who did the immediate work on a given paper, and everybody else alphabetically (similar to 2dFGRS), but also that no-one is automatically put as a co-author on a paper; one must explicitly request co-authorship.

Paczynski (2000) raised the question (in the context of monitoring the whole sky for variability of objects) "should the whole effort be combined in a very large team, with all papers having several dozen authors listed alphabetically, and no way to find out whom to credit and whom to blame for different parts of the project ?".

This problem arises as the big projects involve individuals who worked hard on the instrumentation and data reduction, and they deserve credit for their efforts. However, one alternative would be to have technical papers (or web sites) written by those who contributed to the infra-structure of the project, which will later be quoted by any paper resulting from the survey. The same holds for more scientific aspects of the collaboration, i.e. apart from core papers, to break the publications down into specific studies with the authorship of those who directly contributed. In the case that two (or more) groups within the team attempt to address the same question by analysing the data differently, it seems most logical to simply publish two separate papers. Another possible solution for large collaborative papers is to have authorship by section as well as a global author list of the paper. This would allow a more precise assignment of credit/blame to be apportioned. It will no doubt take some time for the Astronomical community to develop 'rules' regarding authorship and publications.

\section{Public Release, Virtual Observatories and Data Mining}

Time allocation committees oblige survey teams to release the data within a given period. Perhaps the most successful example is that of the Hubble Deep Field (Williams et al. 1996), where the data were made available to all over the WWW, and resulted in a remarkable scientific output by groups not necessarily involved in conducting the survey. We shall no doubt see similar trends with future publically available data sets such as 2dF, SDSS, 2MASS 11 and $6 \mathrm{dF}$ 12. There are in fact plans to establish Virtual Observatories and Astro-grid (distributed CPU) facilities. This is an interesting concept where the data

\footnotetext{
${ }^{11} \mathrm{http}: / /$ www.ipac.caltech.edu/2mass/

${ }^{12}$ http://www.mso.anu.edu.au/ colless/6dF/
} 
produced by large teams go eventually to the individual, and allow small groups to do their own data-mining and analysis. The exact nature of these new digital research facilities still needs to be defined (e.g. Heck 2001). Another aspect of these public domain data is of course that anyone else in world, not only professional Astronomers, can access the data, or at least enjoy some pretty pictures.

\section{Discussion}

In recent years the astronomical community has experienced an enormous growth in the number of projects and photons collected by ground-based and space observatories. This has led to a new style of work in large teams, and competition between big projects. As discussed above, these trends could be positive if the large projects are divided into smaller tasks that allow the individuals (in particular young researchers) to identify their niche. The Astronomical community will have to define rules and ethics related to authorship of papers, and Email and other communication channels in collaborations of tens to hundreds of people. While these issues are occasionally discussed informally, a more open and frank discussion could help to shape the sociology of the new Astronomy.

\section{Acknowledgments}

I thank A. Heck for suggesting to me to write this article, to S. Bridle, A. Bunker, M. Colless, M. Diemling, F. van Leeuwen, J. Nicholas and M. Strauss for providing information and suggestions, and to the participants of the round table tea at the IoA for helpful discussions.

\section{References}

[] Folkes S.J. et al., 1999, MNRAS, 308, 459

[] Heck, A., letter published in the AAS Newsletter, 104 (March 2001).

[] Hoyle, F., Home is where the Wind Blows, 1994, University Science Books, Mill Valley, California.

[] Paczynski, B., 2000, PASP, 112, 1281

[] Peacock et al., 2001, Nature, 410, 169

[] Wallace, P., 1999, The Psychology of the Internet, Cambridge University Press, Cambridge

[] Williams, R.E. et al., 1996, AJ, 112, 1335

[] York, D.G. et al., 2000, AJ, 120, 1588 